\title{
Adrenalectomy improves glycemic responses in congenic obese LA/Ntul//-cp RATS
}

\begin{abstract}
To determine the effects of adrenalectomy on typical insulin-mediated glycemic responses in male obese rats, groups ( $\mathrm{n}=6-12$ rats/phenotype) of normally reared congenic lean and obese animals were fed a Purina chow diet from 6 to 9 weeks of age, and the Chow diet plus a highly palatable cafeteria diet from 9 to 12 weeks of age. The congenic LA/Ntul//-cp rat strain is noted for its longevity and early expression of the obese trait but remains nondiabetic throughout much if not all of its normal lifespan. Subgroups of obese animals were subjected to bilateral adrenalectomy (ADX) at 6 weeks of age to remove glucocorticoid contributions to glycemic parameters. Measures of weight gain (WG )and of glucose tolerance (OGT) were obtained in the three treatment groups at 6, 9 and 12 weeks of age. WG on ADX-obese rats was similar to that of their lean littermates at 6 and 9 weeks of age on the chow diet but increased to twice that observed in their lean littermates from 9 to 12 weeks of age. OGT responses after 30 to 60 minutes and the area under the OGT curve were impaired but not diabetic in obese animals at all ages compared to lean littermates and returned toward those of normally lean rats after ADX. The Insulin to glucose ratio (I:G) was also consistent with insulin resistance in obese but not in ADX-obese or lean rats at 12 weeks of age. In conclusion, ADX resulted in normalization of OGT and glycemic parameters in the obese phenotype at 9 and 12 weeks of age. These results are consistent with normalization of typical insulin-mediated components of glycemic parameters and glucose uptake in peripheral tissues following adrenalectomy of congenic obese rats. The results further suggest that the counterregulatory effects of insulin and glucocorticoid hormones may be contributory to the impaired glycemic responses in the obese phenotype of the LA/N//-cp (corpulent) rat and are consistent with a receptor-mediated element in the development of insulin resistance and glucose uptake in peripheral tissues commonly associated with the early development of obesity in this strain.
\end{abstract}

Keywords: obesity, insulin resistance, metabolomics, overnutrition, rats
Volume 9 Issue 2 - 202I

\author{
Orien L Tulp, Aftab R Awan, George P \\ Einstein \\ University of Science Arts and Technology, Montserrat, BWI, \\ University of Health and Humanities, Virgin Islands (BVI), and \\ the Einstein Medical Institute, Florida USA
}

Correspondence: Orien L Tulp, University of Science Arts and Technology (USAT), University of Health and Humanities, Virgin Islands (BVI), and the Einstein Medical Institute, Florida, South Mayfield Estate Drive, Olveston, Montserrat, BWI, USA, Tel 6I8-650-5372,Email o.tulp@usat.edu Received: November 26, 2021 | Published: December 20,
2021

\section{Introduction}

Insulin resistance is often identified as a significant contributory factor in progression of obesity, non-insulin dependent diabetes mellitus (NIDDM) and their common pathophysiologic sequelae in man and animals, and is rapidly becoming epidemic in proportion in much of Western society. ${ }^{1-3}$ Obesity occurs in the congenic LA/Ntul//$c p$ rat as a result of an autosomal recessive trait and becomes physically apparent in the obese phenotype by 5 to 6 weeks of age by the physical appearance of pre-obese characteristics. ${ }^{4,5}$ Because the only genetic difference between the lean and the obese phenotype in this strain is the multigenerational epigenetic expression of obesity in $25 \%$ of the offspring of breeding pairs that are heterogeneous for the $-c p$ trait, but with all other inherited attributes being equal, the LA/Ntul//-cp strain becomes an excellent model to examine mechanisms which may contribute to the development of obesity and its pathophysiologic sequelae. ${ }^{4}$ The obese phenotype develops elevations of plasma insulin, amylin and impaired glucose tolerance by 12 weeks of age and which persist thereafter in both male and female offspring but both genders remain non-diabetic under normal laboratory conditions throughout all or much of their remaining lifespan, suggesting that the defect in insulin action in the obese phenotype likely resides at the receptor level. ${ }^{5-8}$ Cortisol elevations and other abnormalities in glucorticoid action have been reported in obesity by several authors, where the elevations in adrenal glucocorticoid hormones are presumed to exert a counter-regulatory influence on insulin actions, thereby contributing to the insulin resistance and impaired glucoregulation commonly observed in obesity and NIDDM. ${ }^{2,9}$ Bukowiecki and colleagues demonstrated that insulin resistance also contributed to impaired expression of norepinephrine-stimulated thermogenesis in brown adipose tissue of SHR/N-cp rats, which share the same $(-c p)$ trait for obesity as the LA/Ntul//-cp strain, thereby likely impacting a major physiologic component of non-shivering thermogenesis and energy balance in man and animals. ${ }^{10-15}$

In addition, Tulp and colleagues have previously reported that the obese phenotype of the LA/Ntul//-cp strain also demonstrates impaired thermogenic responses to overnutrition when fed a cafeteria diet and which also resulted in impaired thermogenic responses. DeBolt et al. examined the thermogenic responses in aging female animals of the LA/Ntul //-cp strain and further showed that the impaired thermogenesis persisted for up to 2 years, considered to represent much of the lifespan of this longevity-prone strain. ${ }^{5,8,14,15}$

The LA/Ntul//-cp strain has been shown to demonstrate one of the longest living obese rodent strains yet characterized, with normally fed and reared animals of the lean phenotype surviving up to 4 or more years under standard laboratory conditions, and obese phenotype littermates surviving 3 years or longer. ${ }^{4,5,8,14}$ The parent LA/N strain was noted for its longevity by Hansen and thus selected for the development of the parental LA/N-cp strain. ${ }^{16}$ The $-c p$ trait was bred into the LA/N strain from the Koletsky rat, ${ }^{17}$ and the resulting $\mathrm{LA} / \mathrm{N}-c p$ rats then backcrossed for 12 or more cycles by Hansen to establish the congenic status of the model whereby the only known difference between the obese and the lean phenotypes is the apparent 
epigenetic expression of the $-c p$ trait. ${ }^{16,18}$ The mechanism of the impaired thermogenesis and insulin resistance which now occurs in this strain is likely a contributor to the caloric efficiency and more rapid rate of weight gain in the obese than in the lean phenotype, and may occur due the combined effects of thyroidal, glucocorticoid and sympathetic actions. ${ }^{9-12,19-20}$ The purpose of the present study was to further define the elements of the impaired thermogenesis associated with the epigenetic expression of the $(-c p)$ trait, beginning from an age prior to the expression of overt obese characteristics, to early adulthood and attainment of reproductive age in the lean but not the obese phenotype, which have not been observed to reproduce with any reliably to date for unknown factors but likely due to endocrinerelated functions, as histologic studies revealed the presence of histologically normal appearance of spermatozoa in the testes of obese animals when examined under light and electron microscopy. ${ }^{21}$

While both the lean and the obese phenotypes develop insulin resistance by early adulthood, studies by Michaelis and others have reported that neither phenotype has been found to develop NIDDM throughout much of their lifespan, consistent with an impaired response in insulin action at the receptor level. Because both NIDDM and obesity have a strong familial predisposition in human populations and thus a likely genetic predisposition may contribute to the obesity and its related attributes. However, the expression of obesity and NIDDM parameters may differ in the extent of the molecular mechanisms that contribute to the development of the two disorders. In the parental LA/N-cp and the derivative LA/Ntul// $-c p$ strain the defect in insulin action likely survives as a receptor level entity only, while in the SHR/N-cp strain which shares the same $(-c p)$ trait for obesity, and is expressed in the spontaneously hypertensive rat $\mathrm{x}$ NIH (SHR/N) background, the obese phenotype demonstrates both obesity and NIDDM by early adulthood, likely due to the expression of both a receptor and a post-receptor defect in insulin actions., ${ }^{4,7}$

Thus, the present study was designed to shed further light on the mechanism of expression of obesity in the obese phenotype of the $\mathrm{LA} / \mathrm{Ntul} / /-c p$ strain, and to determine if the counter-regulatory effects of adrenal glucocortical hormones might contribute to the impaired glucoregulatory parameters in this strain.

\section{Materials and methods}

Animal subjects. Male animals were selected from the Drexel colony of LA/Ntul//-cp rats at 6 weeks of age and separated into lean and pre-obese groups based on developing characteristics of the emerging obese state, which reliably included changes in gait, stance, palpable subcutaneous fat and lower resting VO2 in preobese littermates. Selected animals were placed in shoebox cages lined with one inch of pine shavings, in a temperature $\left(22-24^{\circ} \mathrm{C}\right)$ and humidity controlled (50-60\% RH) environment from 6 until 12 weeks of age. Animals were fed Purina Chow \#4012 diet and house water ad libitum throughout the study. The Purina chow had a reported energy density of $3.34 \mathrm{kcal} / \mathrm{gram}$ (manufacturer certificate of analysis) and the detailed composition indicative of a low glycemic index. During the final 3 weeks of the study, when animals were 9 to 12 weeks of age, all animals were offered a highly palatable cafeteria diet ad libitum in addition to the Purina chow ration. Each day the animals were offered 4 different cafeteria food items out of a total of 20 different cafeteria food items.

Daily Caloric Intake. Periodically during the 6-week study, animals were placed in individual metabolic cages for 3 days, and total food consumed measured by weight, corrected for spillage. Animals were fed the Purina chow, ground for easier measurement, from 6 to 12 weeks of age, the duration of the study. In weeks 9 through 12, animals were also offered a highly palatable cafeteria diet in addition to the Purina chow. The cafeteria diet consisted of numerous snack items including cookies, candies, peanut butter cookies and confections, popcorn, hot dogs, bread, etc., all also corrected for spillage daily. The daily caloric intake was computed based information provided on the manufacturer's nutritional information for each individual item consumed. A total of four different food items were offered each day out of 20 different cafeteria food items included in the study diet. Caloric intake reported is the total Purina Chow plus the cafeteria food items.

Resting Metabolic Rates (RMR). Resting metabolic rates were determined in the fasted state in preobese rats with a Collins Small Animal Spirometer apparatus (WE Collins, Braintree MA, USA) fitted with a 1 cubic foot volume sealable Plexiglas animal chamber with constant closed circuit air circulation as described previously. ${ }^{4,8,23} \mathrm{All}$ measures of $\mathrm{VO} 2$ were conducted at thermal neutrality $\left(30^{\circ} \mathrm{C}\right)$, where the temperature was maintained by placement of the animal chamber in a circulating water bath, and corrected for factors of altitude and relative humidity. Data were expressed per $\mathrm{kg}$ of body weight ${ }^{0.75}$ power to correct for potential differences in mass and body surface area in lean vs. obese animals as described by Kleiber and recently re-confirmed by Yang. ${ }^{24,25}$ After an approximate 5-minute period of equilibration, the recording of peak $\mathrm{VO} 2 /$ minute/ $\mathrm{kg}$ body weight $\mathrm{t}^{-0.75}$ was determined after 15 to 30 minutes. Upon initial placement in the chamber, animals were observed to explore their new surroundings for 2 to 3 minutes, after which time they were observed to be resting comfortably for the duration of their occupation in the chamber. In addition, all animals were familiarized and undergone a pretraining experience with the thermogenesis apparatus in an attempt to minimize unnecessary stress prior to being subjected to measurements of $\mathrm{VO} 2$ consumption for record. The metabolizable lean tissue mass of lean and obese animals was similar while the mass of the obese component has been observed to be markedly greater in adult obese vs lean animals of this strain. ${ }^{4}$

Adrenalectomy. Bilateral adrenalectomy (ADX) was performed in pre-obese rats ( $\mathrm{n}=6 \mathrm{rats} /$ group) under pentobarbital anesthesia $(5.5 \mathrm{mg} / \mathrm{kg} \mathrm{BW}$, intraperitoneally) at 6 weeks of age as described by Marchington et al. ${ }^{26} \mathrm{~A}$ single $1.5 \mathrm{~cm}$ mid-posterior incision was made, and the adrenals located by inspection and surgically removed in toto. Care was taken to prevent anesthesia-induced hypothermia by keeping the animals warmed on an electronic heating pad during and after the surgical procedure. Animals were offered a $0.9 \% \mathrm{NaCl}$ solution supplement in addition to house water, ad libitum thereafter following surgery. All animals survived the surgical procedure without outward signs of difficulty or discomfort. At the end of the study, the totality of the adrenalectomy by determined by further dissection to confirm the absence of adrenal tissue remnants.

Oral Glucose Tolerance (OGT), insulin (I), glucose (G) and I:G ratio determinations: Initial and final oral glucose $(G)$ tolerance (OGT) and area under the OGT curve (AUC) and the I:G ratios of rats. Measures of oral glucose tolerance (OGT) were performed in 8 hour fasted animals as outlined by Tulp et al. ${ }^{27}$ Animals were administered a glucose challenge containing $50 \%$ glucose solution via gavage based on body weight ( $25 \mathrm{mg}$ glucose $/ \mathrm{kg} \mathrm{BW}$ ) slowly over a 1-minute duration, and blood obtained via tail-tip vein clipping with a lancet in the fasting state, and after 30-, 60- and 120-minutes post gavage. Bloods were collected in heparinized capillary tubes, capped on one end with putty, centrifuged to separate cells from plasma, and subjected to plasma glucose measurement with a hand-held glucose 
monitor based on the glucose oxidase method. Plasma glucose is recorded and reported as $\mathrm{mg} / \mathrm{dl}$. The area under the OGT curve was calculated as described by Tulp et al. ${ }^{27}$ At the end of the study blood samples were collected in heparinized tubes from the open neck would following decapitation, plasma and cells quickly separated by centrifugation and measures of plasma glucose determined as above and Insulin determined via radioimmunoassay.

\section{Results}

Initial and final body weights of rats. The body weights and mean weights of lean and obese animals at 6,9 and 12 weeks of age are depicted in Figure 1 and Table 1 and show that upon assignment to the respective treatment groups, the body weights of all groups were similar, whilst at the end of the study, the final body weights of the obese and the adrenalectomized obese rats were significantly greater than in the lean phenotype, despite having consumed the same diets and housed under the same laboratory conditions and having the same congenic genetic background except for the $-c p$ trait throughout the study. At 9 weeks of age however, the weights of the adrenalectomized obese rats still remained similar to those of their lean littermates but upon addition of the cafeteria diet option adrenalectomized obese rats developed significant hyperphagia, and their rates of weight gain had matched or surpassed their obese littermates by 12 weeks of age (Figure 2). The mean weight gain between 6 and 9 weeks of age averaged 70 grams in both lean and obese-adx rats, but once the obese + adx group consumed the cafeteria diet, the rate of daily weight gain during the final 3 weeks of the study well exceeded that of the lean phenotype littermates by a factor of 2.75 , similar to a 2.7 fold greater rate of weight gain in the unoperated obese group, and attaining a final rate of weight gain that measured equal to or greater than that observed among the three study groups.

\section{EFFECT OF ADRENALECTOMY AND DIET ON BODY WEIGHT}

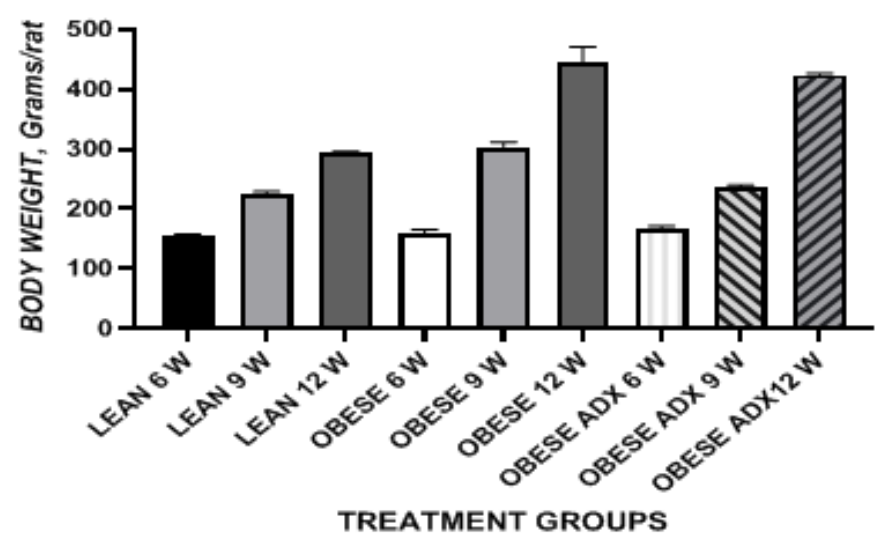

Figure I Data are mean +/- 1 SEM, n= 6 rats/treatment group. Data are grams body weight/rat at 6,9 and 12 weeks of age. Diet at 6 and 9 weeks of age was Purina Chow diet; Diet at 12 weeks of age as Chow + Café.

\section{EFFECT OF ADX ON OGT IN OBESE RATS 6 WKS OF AGE}

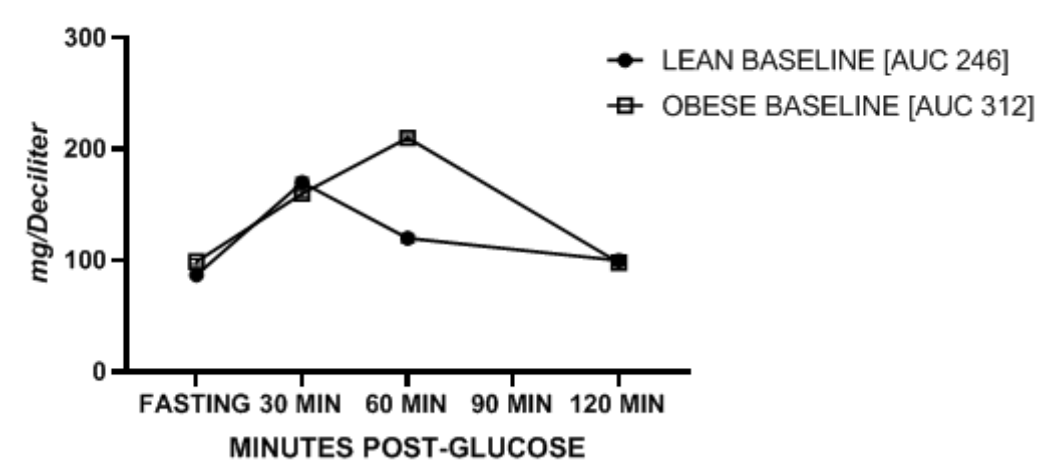

Figure 2 Effect of obese phenotype on OGT and AUC in lean and pre-obese rats at 6 weeks of age. Data are mean $+/-$ I SEM, n=6-12 rats / group. Data for obese rats are significantly greater than lean group at +60 minutes $(p=<0.05)$.

Table I Effect of adrenalectomy and diet on weight gain of lean and obese rats

\begin{tabular}{lllll}
\hline Group & $\mathbf{n}$ & Weight gain, chow & weight gain, chow+café & Total weight gain \\
\hline Lean & 6 & $69.83 \pm 7.96 \mathrm{a}$ & $67.00 \pm 2.92 \mathrm{a}$ & $136.83 \pm 7.96 \mathrm{a}$ \\
Obese & 6 & $145.6 \pm 20.63 \mathrm{~b}$ & $124.67 \pm 34.4 \mathrm{lb}$ & $276.13 \pm 7.52 \mathrm{~b}$ \\
Obese-ADX & 6 & $70.02 \pm 8.96 \mathrm{a}$ & $177.33 \pm 6.36 \mathrm{~b}$ & $238.37 \pm 7.52 \mathrm{~b}$ \\
\hline
\end{tabular}

Table I Rates of weight gain in lean, obese, and obese-adrenalectomized animals fed chow vs. chow+café diets. Data are mean \pm I SEM, $n=6$ rats/group, grams/rat. Groups with different letter superscript are Student-Newman-Keuls application for identification of significantly different subgroups at $p=<0.05$ 
Measures of Oral Glucose tolerance. Measures of oral glucose tolerance and the AUC are depicted in Figures 2-4 and show that the glycemic responses including the AUC of obese rats at 6 weeks of age indicate a prolonged absorptive phase and higher peak blood glucose concentrations than were observed in the lean phenotype, despite being littermates and consuming the same diet, rearing, and housing conditions since birth (Figure 2). The glycemic responses of the three groups of rats at 9 weeks of age are depicted in Figure 3 and show that the glycemic responses including the AUC of obeseadx animals remained similar to those of the lean littermates. While the glycemic responses of the unoperated obese phenotype show moderate glycemic impairment as demonstrated at the younger age. The OGT responses and AUC of the three treatment groups after the cafeteria diet supplement at 12 weeks of age are depicted in Figure 4 and indicate that the OGT responses of the lean and adrenalectomized obese animals remain similar, while the unoperated obese rats continue to reflect modest glucose intolerance but not at a magnitude deemed sufficient to indicate NIDDM. Not shown, random urine glucose measurements were negative in all groups throughout the study.

\section{EFFECT OF ADX ON OGT AND AUC AT 9 WEEKS AGE}

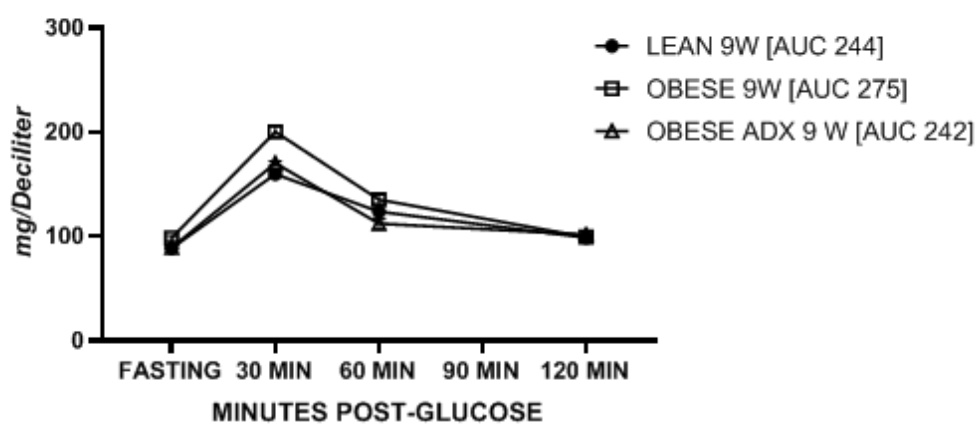

Figure 3 Effect of adrenalectomy on OGT and AUC at 9 weeks of age. Data are mean +/- I SEM, n=6 rats / group. Data for obese rats are significantly greater than other groups at +30 minutes $(p=<0.05)$.

The effects of obesity and ADX+obesity is depicted in Figure 4 and show that the glycemic responses and AUC of the lean and obeseadx groups remain similar and well within the normal range, while the OGT and AUC of unoperated obese rats continue to demonstrate modest glycemic impairment with peak glucose responses significantly elevated in excess of $200 \mathrm{mg} / \mathrm{dl}$. at the 30 -minute time point, but returning to fasting, pre-glucose administration ranges by 120 minutes post glucose ingestion in all groups.

\section{EFFECT OF ADX ON OGT IN OBESE RATS 12 WK OF AGE}

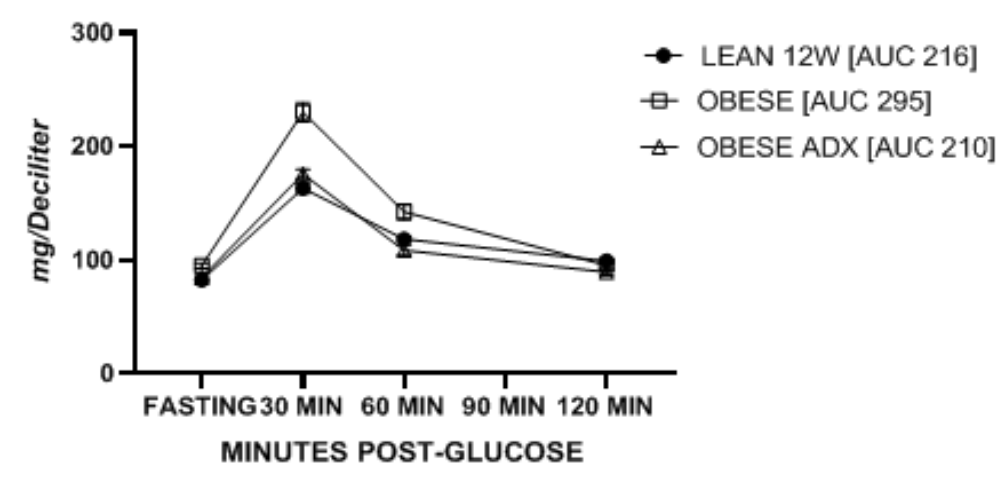

Figure 4 Effect of adrenalectomy on OGT and AUC at 12 weeks of age. Data are mean $+/-$ I SEM, n=6 rats / group. Obese rats were significantly greater than the other groups at +30 and +60 minutes $(p=<0.05)$.

Table 2 Effects of adrenalectomy on final glycemic parameters in lean and obese LA/Ntul//-cp rats

\begin{tabular}{lllllll}
\hline Group & $\mathbf{n}$ & Glucose & Insulin & I:G ratio & AT Mass, g. & $\%$ Adiposity \\
\hline Lean & 6 & $144.0 \pm 9.0 \mathrm{a}$ & $253.3 \pm 20.1 \mathrm{a}$ & $1.43 \pm 0.16 \mathrm{a}$ & $5.54 \pm 0.1 \mathrm{la}$ & $1.87 \pm 0.04 \mathrm{e}-2 \mathrm{a}$ \\
Obese & 6 & $145.5 \pm 15.7 \mathrm{a}$ & $874.0 \pm 30.0 \mathrm{~b}$ & $6.01 \pm 1.40 \mathrm{c}$ & $2 \mathrm{I} .16 \pm 2.10 \mathrm{c}$ & $4.74 \pm 0.47 \mathrm{e}-2 \mathrm{c}$ \\
Obese-ADX & 6 & $126.3 \pm 4.9 \mathrm{a}$ & $299.5 \pm 7 \mathrm{I} .5 \mathrm{a}$ & $2.40 \pm 1.08 \mathrm{a}, \mathrm{b}$ & $12.8 \mathrm{I} \pm 2.30 \mathrm{~b}$ & $3.02 \pm 0.54 \mathrm{e}-2 \mathrm{~b}$ \\
ANOVA & & $\mathrm{ns}$ & $\mathrm{P}=<0.05$ & $\mathrm{P}=<0.05$ & $\mathrm{P}=<0.05$ & $\mathrm{P}=0.05$ \\
\hline
\end{tabular}

Data are mean +/- I SEM, $n=6$ rats/group. Bloods collected from the open neck wound following sacrifice by decapitation in a semi-fasted state. G. The AT mass is the arithmetic sum of Dorsal, Retroperitoneal and Epididymal fat depots at 12 weeks of age; \% Adiposity is arithmetic sum of $g$ Dorsal+Retrperitoneal+Epididymal mass / g. BW $\times 100$. Groups with different letters in superscript indicate distinct groups by Student-Neuman-Keuls application for identification of significantly different subgroups 
Relative adiposity levels are also depicted in Table 1, with the adiposity in the right column and the I:G ratios in the central column. These findings demonstrate a striking similarity in their pattern, with both obese and ADX-obese groups having greater net adiposity than their lean littermates, with the obese and obese-adx groups being greater than their lean littermates despite having the same genetic origins and consuming the same diet and environment since birth. In contrast, plasma insulin levels were greatest in the obese, intermediate in the obese-adx, and lowest in the lean littermates. The apparent disparity in adiposity and I:G ratios in the obese+ADX group is of interest, as it more than likely is reflective of the similar magnitude of hyperphagia observed in the ADX-obese group in the absence of counterregulatory impact of glucocorticoid hormones and resulting improvement in insulin sensitivity.

\section{Discussion}

In rat skeletal muscle, Kahn reported that glucocorticoid actions can inhibit the insulin stimulated translocation of GLUT4 and other glucose transporters from their intracellular compartment to the plasma membrane in response in muscle, thereby contributing to or inducing a rebound increase in insulin concentrations to effect peripheral glucose uptake and corresponding to progressive increases in the magnitude of insulin resistance. This inhibition of insulin dependent GLUT4 transporter actions likely results in the progressive development of insulin resistance via greater levels of insulin secretion. In human adipose tissue, glucocorticoids also induce adipocyte differentiation leading to increased adiposity and which may further exaggerate or exacerbate the development and magnitude of insulin resistance. ${ }^{28-30}$ Thus, removing the inhibitory adrenal-glucorticoid influence on the GLUT4 mediated glucose transport would be expected to have a permissive if not stimulatory effect on insulin stimulated GLUT4 translocation from the endoplasmic reticulum to the outer cell membrane, and increase the capacity for peripheral glucose uptake in peripheral tissues. In addition, insulin is known to stimulate numerous activities in affecting energy metabolism adipose and other peripheral tissues, including insulin stimulated lipogenesis, preadipocyte differentiation and lipid accretion, and development of the attendant adiposity. In the present study, glycemic parameters including the progressive development of insulin resistance were modestly increased with age in the obese phenotype and were already apparent by 6 weeks of age and increased further with the cafeteria diet in the obese phenotype by 12 weeks of age. In contrast, insulin resistance as indicated by the insulin to glucose ratio and the AUC decreased following adrenalectomy in obese rats when consuming the chow diet, but when the adrenalectomized animals consumed the hyperphagia-inducing cafeteria diet in addition to the chow diet, they developed adiposity at an accelerated rate without the same magnitude of elevations observed in the insulin to glucose ratio or the AUC. Their rate of weight gain surpassed the rate of weight gain and adiposity of non-adrenalectomized obese littermates, despite the absence of glucocorticoid hormones due to their prior adrenalectomy. The hyperphagia and subsequent rapid weight gain after introduction of the cafeteria diet in adrenalectomized animals was also remarkable, consistent with an enhanced propensity for glucose uptake in peripheral tissues, lipogenesis, and subsequent energy deposition. Insulin to glucose ratios were significantly increased only in the obese phenotype after the cafeteria feeding regimen, but the I:G ratios in the obese-adx group were consistent with enhanced substrate uptake in peripheral tissues, consistent with an apparent likely increase in insulin stimulated translocation of GLUT4 transporters, thereby enabling more efficient energy deposition in both fatty and lean tissues, and enabling optimal growth in both lean and fatty compartments. These observations are consistent with a receptor mediated process for glucose uptake and disposal, which was impaired in the obese phenotype but modifiable by the level of caloric intake when consuming the cafeteria diet.

The initial phase of the plasma glucose concentrations of the OGT between fasting and 30 minutes post gavage glucose administration is taken to represent the luminal absorption and initial peripheral distribution and uptake in peripheral tissues as represented in the first phase of the OGT curve. Luminal absorption of glucose at this stage in the absence of other nutrients or fiber is typically $100 \%$ efficient as luminal glucose uptake does not require further oxidation and occurs in the absence of insulin. The second phase of the OGT curve is represented by plasma glucose levels beyond the 30-minute mark, represented in this study during time points from +30 to 120 minutes post-gavage at which time plasma glucose concentrations normally return to pre-gavage levels in the absence of NIDDM or severe glucose intolerance by 120 minutes of the glucose challenge. Thus, this post absorptive phase represents plasma glucose distribution and uptake in peripheral tissues and can reflect the presence of insulin resistance. The insulin resistance may also reflect an impairment or dysregulation of the insulin dependent GLUT4 translocation process in muscle and adipose tissues, but which can occur without insulin mediation in liver and nervous tissues. Thus, the glucose concentrations were similar at +30 minutes in all groups, as luminal absorption in the gastrointestinal tract occurs rapidly and completely under fasting conditions and were unlikely to have been impacted by other gastrointestinal factors, and the luminal absorption process does not require the presence or actions of insulin. Once the absorbed glucose becomes located in the plasma, most of the remaining peripheral tissues including the lean body mass and adipose tissue require the presence of an effective and efficient insulin-receptor interface, and includes the translocation of GLUT receptors in muscle and adipose tissue, from the intracellular endoplasmic reticulum of the cell to the outer plasma membrane, where the GLUT4 transporters can now quickly and efficiently translocate the glucose moieties from the plasma membrane to the interior of the cell where it can readily contribute to glycogen formation or to undergo oxidation via glycolysis and provide substrates for multiple cellular functions including lipogenesis, hexose monophosphate shunt, and other cellular biochemical processes. The peripheral uptake of glucose, as indicated in the OGT curves of ADX-obese rats appears to have occurred similarly to those of their nonobese lean littermates consuming the same dietary constituents, albeit with lesser magnitude of hyperphagia among the lean littermates, thus consuming different total quantities of the diet. In previous studies, when obese rats were pair-fed to their lean littermates, they still became obese at an intermediate magnitude however, suggesting that caloric intake per se is not the main reason the obese phenotype develops obesity beginning at an early age but that differences in the caloric efficiency of weight gain do contribute to the early epigenetic expression of the obese phenotype. ${ }^{5}$

The magnitude of insulin resistance which developed in the obese phenotype resulted in delayed glucose uptake in those peripheral tissues such as muscle and adipose tissue where insulin mediated GLUT4 glucose uptake occurs. In contrast, in liver and nervous tissue and in the luminal absorptive surface of the gastrointestinal track, glucose uptake would be expected to occur unimpeded but over a longer duration after feeding in non-luminal tissues, thereby contributing to the greater AUC observed in the OGT of obese animals. These observations are consistent with a progressive development of a receptor level impairment in glucose uptake secondary to uncomplicated insulin resistance, and which may include glucocorticoid linked delayed cytoplasmic translocation of GLUT4 
receptors necessary for efficient glucose uptake in insulin sensitive tissues. In non-insulin sensitive tissues plasma glucose uptake likely occurred normally.

Many factors including stress and diet may also contribute to aberrations in glucocorticoid actions in peripheral tissues. Like other steroid hormones, glucocorticoid hormones are lipid soluble and can enter cells via diffusion, and become transported throughout the cell, and where they may eventually impact on nuclear chromatin receptors to effect and enable a broad range of mRNA-linked metabolic actions, including but not limited to an inhibition of translocation of GLUT4 glucose transporters from the endoplasmic reticulum to the plasma membrane. Removal of the glucocorticoid influence would be expected to restore membrane associated factors of glucose uptake including GLUT4 translocation events in insulin dependent peripheral tissues, and a restoration or enhancement of glucose and energy homeostasis. The modestly greater insulin concentrations observed in ADX-obese rats would be expected to result in a greater efficiency of glucose uptake in peripheral tissues and enable greater substrate availability for energy deposition in adipose tissues. Thus, the results of this study further suggest that the counterregulatory effects of insulin and glucocorticoid hormones may be significant contributory factors in the impaired glycemic responses observed in the obese phenotype of the LA/N//-cp (corpulent) rat and are consistent with a receptor-mediated element in the development of insulin resistance and glucose uptake in peripheral tissues as has been commonly associated with the early development of obesity in this strain.

\section{Acknowledgments}

The author wishes to acknowledge the contributions of Dr. Susan DeBolt for data collection, and Dr. Stephen Dubin, Mr. Huang Peisong and Ms. Mary Victor of Drexel University for veterinary and animal care support and the late Dr. Otho E Michaelis IV, of the Carbohydrate Nutrition Research Laboratory, Beltsville, MD, USDA for contributions, data collection and management assistance in completion of this research.

\section{Funding}

None.

\section{Conflicts of interest}

The authors declare no conflict of interest.

\section{References}

1. Jacks DG, Kerna NA. A comprehensive analysis of obesity: Part 1: Overview of Obesity. J Obes Nutr Disord. 2018.

2. Poku-Mensah, C, George P Einstein, GP and Tulp, OL. Insulin sensitivity is a significant influential risk factor in the development of cardiovascular disease in diabetes mellitus. Faseb J 35, 2021

3. Center for disease control and prevention. National Health and Nutrition Survey. (NHANES Data Base, NIH), 2013-2014.

4. Tulp OL. Characteristics of thermogenesis, obesity, and longevity in the LA/N-cp rat. ILAR News Journal. 1990:32(3):I33-I39.

5. Tulp OL. Effects of aging, phenotype, and carbohydrate feeding on caloric efficiency and adiposity in the LA/Ntul//-cp rat. Adv Obes Weight Manag Control. 2021:11(1):5-11.

6. Huang HJ, Young, AA, Koda JE, et al. Hyperamylinemia, hyperinsulinemia, and insulin resistance in genetically obese LA/N-cp rats. Hypertension. 1992:19(1 Suppl):101-109.
7. Michaelis OE IV. New models of genetically obese rats for studies in diabetes, heart disease, and complications of obesity, veterinary resources branch, division of research services, NIH publication, Bethesda, MD. 1988:13-15.

8. Tulp OL, Einstein GP. Thermogenesis, aging and obesity in the LA/Ntul//cp (corpulent) rat. Adv Obes Weight Manag Control. 2021;11(2):37-43.

9. Kahn, CR. Role of insulin receptors in insulin-resistant states. Metabolism 1980;29(5):455-466.

10. Bukowiecki LJ, Deshaies, Y, Collet, AJ, et al. Major thermogenic defect associated with insulin resistance in brown adipose tissue of obesediabetic SHR/N -cp rats. Am J Physiol. 1991;261(Endo Metab):E204 E213.

11. Marette A, Tulp OL, Bukowiecki LJ. Mechanism linking insulin resistance to defective thermogenesis in brown adipose tissue of obese diabetic SHR/N -cp rats. Int J Obese. 1991;15:823-831.

12. Atgie C, Marette A, Desaultels M, et al. Specific decrease in mitochondrial thermogenic capacity in brown adipose tissue of obese SHR/N-cp rats. Am J Physiol. 1993;265(Cell Physiol 34):C1674-C1680.

13. Tulp OL. The effects of experimental over nutrition on non-shivering thermogenesis and obesity in LA/N-cp rats. Comp Bloch Physiology. 1991;98A:567-574

14. Tulp OL, DeBolt SP. Aging and obesity in the corpulent rat. Nestle Nutrition Series, Research and Practice in MNA and Aging. 1999:149155.

15. DeBolt, S.P. Effects of aging and obesity on adaptive thermogenesis and energy metabolism in LA/N-corpulent rats. PhD Thesis, Drexel University, 1992. 314 pp.

16. Hansen CT. The development of the SHR/N- and LA/N-cp (Corpulent) congenic rat strains. New models of genetically obese rats for studies in diabetes, heart disease, and complications of obesity. NIH publication, Division of Research Services, Veterinary Resources Branch, National Institutes of Health, Bethesda, MD. 1988:7-10.

17. Koletsky, S. Obese spontaneously hypertensive rats-A model for the study of atheroschlerosis. Exp Biol Pathol. 1973;19:53-60.

18. Greenhouse DD, Hansen CT, Michaelis OE. Development of fatty and corpulent rat strains. ILAR News Journal. 1990;32(3):2-4.

19. Wolpert SI, RM Bye, OL Tulp. Effect of $\alpha$-methylparatyrosine on thermogenesis in cafeteria fed rats. Clin Res. 1983;31(3):677A.

20. Wolpert SI, RM Bye, OL Tulp. Effect of $\alpha$-methylparatyrosine on thermogenesis before and after cafeteria feeding in rats. Fed Proc. $1984 ; 43(5)$

21. Sasner JM, CT Jones, GD Marco, et al. Morphometric features of proteinrestricted LA/N-cp (corpulent) rats. Prog. Colby-Bates-Bowdoin boil. Research symposium. 1983.

22. Michaelis OE, Ellwood KC, Tulp OL, et al. Effect of feeding sucrose or starch diets on parameters of glucose tolerance in the LA/N-corpulent rat. Nutr Res. 1986;6(2):95-99.

23. Young NL,d Tulp OL. The effects of norepinephrine and nutritional status on resting metabolic rates in LA/N-cp rats. Comp Biochem Physiol. 1989;94(4):597-602.

24. Kleiber M. The fire of life: an introduction to animal energetics. New York NY USA: Wiley publishers; 1961.

25. Wang ZM, Zhang J, Ying Z, et al. Organ-tissue level model of resting energy expenditure across mammals: new insights into Kleiber's law. Int Scholarly Research Network, IBSN Zoology. 2012.

26. Marchington D, Rothwell NJ, Stock MJ, et al. Energy balance, diet induced thermogenesis and brown adipose tissue in obese (fa/fa) Zucker rats after adrenalectomy. $J$ Nutr. 1983;113:1395-1400. 
27. Tulp OL, Brown T. Effect of a fructan-chromium complex on glycemic responses of congenic obese LA/Ntul//-cp rats. J Nutr Health Food Eng. 2016;5(2):594-598.

28. Dimitriadis G, Leighton B, Parry-Billings M, et al. Effects of glucocorticoid excess on the sensitivity of glucose transport and metabolism to insulin in rat skeletal muscle. Biochem Jj. 1997;321:707-712.
29. Gathercole LL, Morgan SA, Bujalska IJ, et al. Regulation of lipogenesis by glucocorticoids and insulin in human adipose tissue. PLOS ONE. 2011;6:e26223.

30. Hauner H, Schmidt P, Pfeiffer EF. Glucorticoids and insulin promote the differentiation of human adipocyte precursor cells into fat cells. $J$ Clin Endocrinol Metab. 1987;64:832-835. 